\title{
Biological inspired algorithm for Storage Area Networks (ACOSAN)
}

\author{
Anabel Fraga Vázquez ${ }^{1}$ \\ 1 Universidad Carlos III de Madrid \\ Av. Universidad, 30, Leganés, Madrid, SPAIN afraga@inf.uc3m.es
}

\begin{abstract}
The routing algorithms like Storage Area Networks (SAN) algorithms are actually deterministic algorithms, but they may become heuristics or probabilistic just because of applying biological inspired algorithms like Ant Colony Optimization (ACO) of Dorigo. A variant suggested by Navarro and Sinclair in the University of Essex in UK, it is called MACO and it may open new paths for adapting routing algorithms to changes in the environment of any network. A new algorithm is anticipated in this paper to be applied in routing algorithms for SAN Fibre Channel switches, it is called ACOSAN.
\end{abstract}

\section{INTRODUCTION}

This paper helps to create new paths for betters routing algorithms based in biological inspired algorithms like ACO (Ant Colony Optimization) of Dorigo $[5,18,19]$. The base of the paper is to apply these kind of algorithms and variants of that in Fibre Channel's switches (FC), for package routing in an adaptive way. In particular the Multiple Ant Colony Optimization of Navarro and Sinclair and ANTNET of Dorigo are very useful in that matter $[11,13,18]$.

The reminder of this section establishes the history and introduction to the SAN networks, and benefits of this technology. Section 2 explains some key concepts and problems of Fibre Channel networks. Section 3 surveys some related previous work applying ACO to networking problems. Sections 4 describe basis for the algorithms proposed and the algorithm itself. And the final sections cover acknowledgments, future work and conclusions.

\subsection{History and definitions}

The increasing need year by year to connect disks to computers by SCSI connectors, which is a standard in the eighties in parallel connections, but not so fast as expected because of the problem that parallel connection have in front of serial connections.

Please use the following format when citing this chapter:

Vázquez, A.F., 2006, in IFIP International Federation for Information Processing, Volume 217, Artificial Intelligence in Theory and Practice, ed. M. Bramer, (Boston: Springer), pp. 109-118. 
Day by day new faster connections are needed and technology does not stop and must not. The Fibre Channel technology is a prove of advance, it starts in the nineties and it has an appreciated speed which is over Gigabits, serial connection, and allows large distances over 10 kilometers.

The external storage is a new discovers, disks not connected anymore point-topoint to servers. Large storage arrays of disks now are the centre of the external storage, it may content five disks or even thousands of disks depending on the size of the company and the data to be storage, terabytes of information are connected by Fibre Channel to servers.

Brocade, an enterprise recognized in the area of Fibre Channel' s switches, is at the moment one of the factories for Fabric topologies in Storage Area Networks (SAN). This company defines SAN as a network for storage and system components, which are all communicated in a Fibre Channel net, used to consolidate and share information, offering high performance links, high availability links, higher speed backups, and support for clustering servers.

\subsection{Providers}

The main providers of SAN and Fibre Channel spares are placed in Table I. McData is the growing company in the area, followed by Brocade. McData works with IBM and Brocade works with Hewlett Packard (HP). These alliances are strategic for both companies in order to provide a whole package for the customers.

Table 1. Fibre Channel's switches providers

\begin{tabular}{clc}
\hline Rank & Provider & Growing rate (\%) \\
\hline 1 & McData (IBM) & 17 \\
2 & Brocade (HP) & 5 \\
3 & Cisco Systems & 36 \\
4 & CNT/Inrange & 8
\end{tabular}

Cisco is showed as one of the companies with the grater rate of growing but not only for SAN technology, the company is in a privileged position because of the large quantity of switches for any kind of network.

\subsection{Structure and topology of SAN networks}

There are different topologies for storage networks, the three basics are showed in Figures 1, 2 and 3. Figure 1 shows a point-to-point network with SCSI technology. 
Figure 2 shows a Fibre Channel technology based network which covers over ten kilometres. And finally, Figure 3 shows a typical SAN network of Fabric typology.

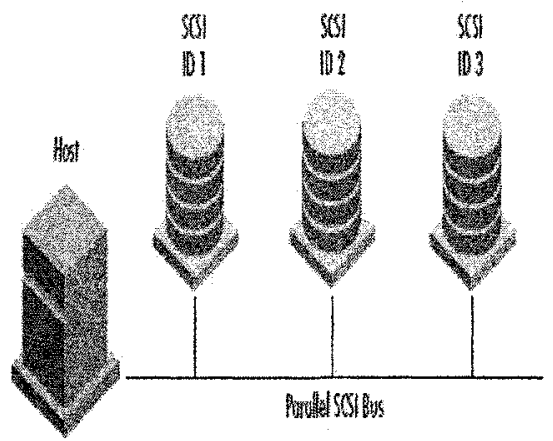

Fig. 1. Basic SCSI technology net between disks and server.

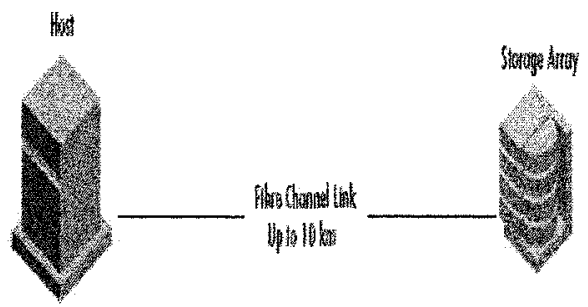

Fig. 2. Fibre Channel net for over 10 kilometres.

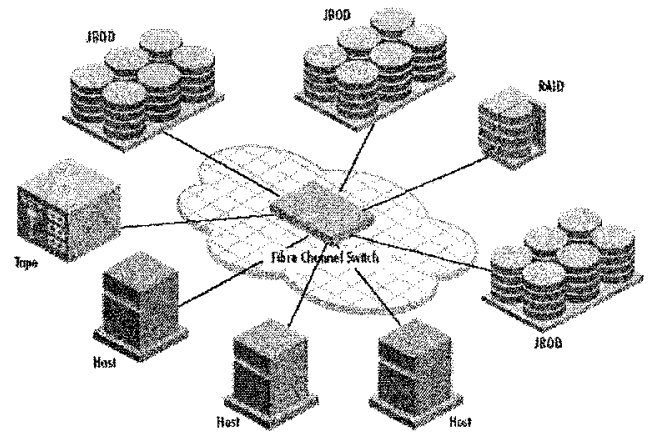

Fig. 3. Typical Fibre Channel network in multi-servers environment. 


\subsection{Benefits}

Storage Area Networks (SAN) guarantee high availability in the nodes interconnected, allows consolidating information in disk arrays able to storage thousands of bits or bytes in a single point of storage. An additional advantage is to reduce network overload originated for automatic backups. Then again, allows to accelerate information access speed and makes certain fault tolerance. In case of a physical disaster, the availability that servers have because of been placed in different locations connected to the storage network makes possible to maintain unaffected the operation of a company.

\section{FIBRE CHANNEL}

Fibre Channel technology is a generic mechanism that allows high speed of transfers in long distances. It has physical definitions in the physical layer because of the transport protocol, like OSI for TCP/IP, the protocol tolerates TCP/IP and SCSI interfaces.

In general, it is easy to provide routing algorithms for Fibre Channel, but they are not specified or even public in the switches architecture provided. For example José Duato [12] published a BFS algorithm for routing SAN networks with Fibre Channel. But biological inspired algorithms are missing, and they could be an improvement for routing and adapting to networks exclusive of need for redefine algorithms or nodes in a switch.

Fredman, DataCore employee, defines Fibre Channel as a technology based on standards, innovative, functional to replace SCSI connections between disks, backup robots and servers.

Fibre Channel topologies are three:

1. Point-to-point: Based on simple links of connection between disks and servers.

2. Arbitrated Loop (FC-AL): Based on Hubs integrated to the net for routing packages.

3. Fabric: Based on switched network for routing packages.

Fibre Channel uses a communication protocol analogous to OSI with seven layers, similar to TCP/IP. The layers in the protocol are showed in Figure4.

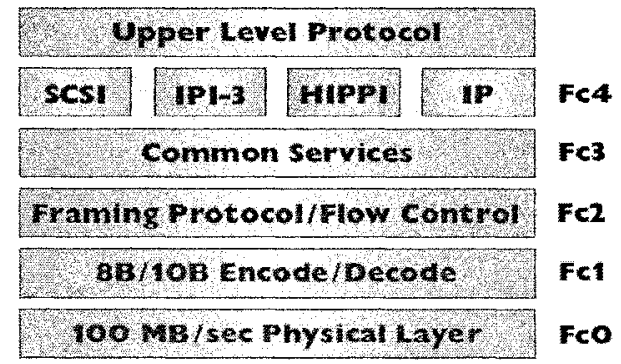

Fig. 4. Fibre Channel's protocol, similar to OSI model. 


\section{USES OF ANT COLONY OPTIMIZATION IN ROUTING ALGORITHMS}

Marco Dorigo $[6,18,19]$, the father of an special biological inspired algorithms based on studies of ants natural environments and behaviour: Ant Colony Optimization (ACO) and ANTNET. In general, ants leave pheromone trail in the way to the nest and the food location. So movements are based in quantities of pheromone in the paths to follow. As much pheromone located in one path then higher will be the probability to go in the course of that way. There are always of course ants that not follow the most probable path for obtaining new sources of provisions.
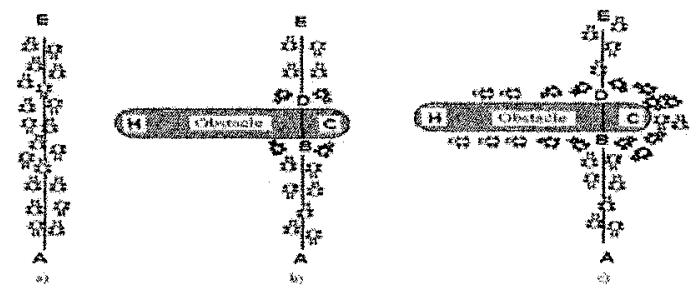

Fig. 5. Ants travelling from nest to food and suddenly an obstacle appears (Taken

from Dorigo's paper explanation of ACO algorithms).

As a general rule, an heuristic function is involved in the movement of the ants in the natural environment where they are located. If in the way between nest and provisions suddenly appears an obstacle as showed in Figure 5 [13], then ants will generate a new trail and the shortest path will be used naturally because the pheromone trail is strongest in the short path for moving from nest to food and so on. This phenomenon occurs because more ants travel for the short path than for the long one. In case of two paths or more available and two shorts paths are accessible then a balance of ants traveling on them will be observed (Figure 6) [13].
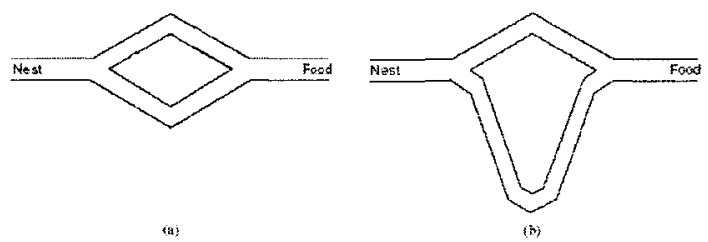

Fig. 6. More than one optimum path from nest to food.

The load balance of ants is showed in Figure 7 [13]. The first chart shows the case "a" of Figure 7 and the second chart shows the case "b" of that figure.

Dorigo [6] established an algorithm for pheromone evaporation, it occurs naturally, if not the search for new food would not be possible. 


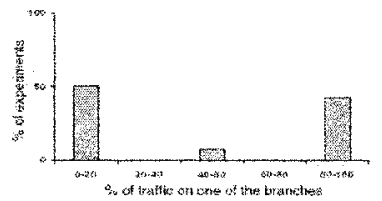

(n)

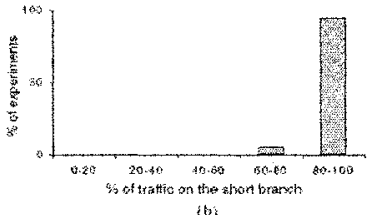

Fig. 7. Load balance of ants with more than one path possible $[6,18,19]$.

\section{ROUTING ALGORITHM}

This section shows the basis and the algorithm itself.

\subsection{Basis}

MACO is an algorithm based in the ACO algorithm; it was originated in Essex University in UK. It was proposed for load balancing and routing in networks. It uses different ant colonies with different pheromones types, so ants of one colony will be repelled by others' pheromones and attracted by owns pheromones. It is an innovative approach and useful.

It has three kinds of algorithms. The first, it is based in local update of pheromone values. The second, it is based in update of pheromone values depending on distance. And the third, based in a global update of pheromone values depending on quantity of ants going on one path [13].

By this means, avoiding the problem of down nodes or faults in the network are interesting for adaptive environment algorithms.

\subsection{Algorithm for storage area networks}

Thanks to the MACO algorithm [13] and ANTNET [18] as a basic idea, is possible to postulate an algorithm called ACOSAN for Fibre Channel's switches as follows:

1. It is used more than one ant colony in order to produce different kinds of pheromones with the intention to find different optimal paths of routing for load balance purposes. A routing table will be filled with the information of routing between nodes of the net in the switch.

2. There must exist a first full filled routing table, it must be initialized using a deterministic algorithm, a manual default configuration or an existing table with default values of pheromone. It must be done in order to avoid the initial delay for generating the first version of that table.

3. Cycles of ant colonies will circumnavigate the network of switches and routing tables will be updated with new values of pheromones. It will allow maintaining distributed tables all the time. 
4. The repelling mechanism will help ants to go over different routes, so load balancing routes could be generated.

5. In each cycle ants moves from every start and end possible at each point. The selection of links is stochastic, it depends of level of repelling and attraction of pheromones by ants, it is defined in the probabilistic function which is explained in the mathematical bases of the algorithm.

6. Once a loop is detected and no new version of the table is generated after a number finite of cycles, the ants are destroyed.

7. In the moment all ants are destroyed then a new cycle is placed after a variable of time defined in the algorithm in order to find better paths of routing in case of environmental changes in the links.

8. Ants must update pheromone trails in each cycle in local (on every switch). And trail must be updated as well after a cycle ends depending on global distances of paths and pheromone amounts in each path.

9. The pheromones are evaporated in each cycle as in the real life, if not new paths would not be found.

10. The best path in each cycle is stored for generate a final routing table of the cycle.

This algorithm can be applied in generic networks on top. But Fibre Channel is the primary target.

\subsection{Mathematical bases for routing algorithm}

The mathematical bases for the algorithm proposed are clearly established in Dorigo's papers $[3,4,5,6]$ and Navarro and Sinclair's paper [13] and they are applied to this algorithm also, as shown in principle 1 to 7 .

The algorithm will be able to control attraction of pheromones using formula (1), where is the weight of attraction for a link $k$ by ant $j$, and will be the sum of pheromone over all links available, the ant must select one, where is the set of all links available for ant $\mathbf{j}$ depending on the position in the net.

$$
\alpha_{k j}=\frac{p_{k j}}{\sum_{i \in A_{j}}\left(P_{i j}\right)}
$$

The repelling formula for pheromones by each ant will be updated globally conditional on the amount of ants in each link (3), it is the best result as suggested by Navarro and Sinclair [13], where (2) represents the use of the link k, by total sum of ants of each types crossing the link $\mathrm{k}$. But, is evaporated as pheromone at the end of each cycle represents the constant of evaporation.

$$
u_{k}^{t+1}=\rho \bullet u_{k}^{t}\left(\forall k, t=\sum_{i=1}^{T} S_{i}\right)
$$




$$
\beta_{k j}=\frac{u_{k}}{\sum_{i \in A_{j}}\left(u_{i}\right)}
$$

The probability function for movement of an ant $\mathrm{j}$ for taking link $\mathrm{k}$ will be (4) represented by where is an important constant defining the weight that repelling will have for a different kind of pheromone found in the journey. Dividing by is sure that probability will be improved as much as attraction is greater, and diminish as much as repelling grows.

$$
\gamma_{k j}=\frac{\alpha_{k j} / \beta_{k j}^{r}}{\sum_{i \in A_{j}}\left(\alpha_{i j} / \beta_{i j}^{r}\right)}
$$

The pheromones' update rule is showed in equation (5) without evaporation, used by Coloni $[3,4,5]$ where $Q$ is the amount of pheromone placed by an ant when passing thought a link, and evaporation reduce in the amount of pheromone placed for each link, it is denominated evaporation factor (6), where T represents each cycle of the algorithm.

$$
\begin{aligned}
& p_{k j}^{t+1}=p_{k j}^{t}+Q \\
& p_{k j}^{t+1}=\rho \cdot p_{k j}^{t}\left(\forall j, \forall k, t=\sum_{i=1}^{r} S_{i}\right)
\end{aligned}
$$

If global update is considered by distance and not by amount of ants in each link, then equation for pheromone update will be (7) and factor would not be used, where will be the distance of link $k$, in route followed by an ant $j$.

$$
p_{k j}^{t+1}=p_{k j}^{t}+Q / L_{j}^{T}\left(\forall j, \forall k \in R_{j}^{T}, t=\sum_{i=1}^{T} S_{i}\right)
$$

\subsection{Probabilistic vs. Deterministic}

Analyzing deterministic and probabilistic algorithms in references [13], as shown in Figure 8 , in general a heuristic algorithm will converge as a deterministic in similar solutions, but a gap is in the beginning of the algorithms. That is the reason to use a deterministic initial routing table for avoiding this gap at the beginning.

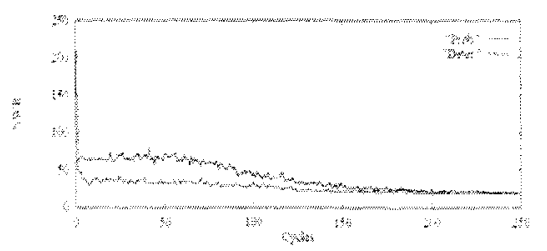

Fig. 8. Probabilistic/deterministic algorithms' general performance [13]. 


\section{CONCLUSIONS AND FUTURE WORKS}

An algorithm for routing in adaptive environments is shown in the paper using biological inspired algorithms like Ant Colony Optimization. It will help to detect changes in the network and overload in some nodes. In general, evolving algorithms are useful to solve these problems.

The need of an ACO algorithm for networks was touched by Dorigo $[18,19]$ in the ANTNET seminal work in 1997, but applying those techniques to Fibre Channel networks for SAN are novel, it could be a preliminary approach for adapting such kind of algorithms into this technology.

ACO, MACO and new algorithms as shown in the paper are able to adapt to the environment without need of human interaction in case of failure.

Ants in the network are not a problem for high speed networks like Fibre Channel switches, if the problem of changing environments is solved by this kind of algorithms [9].

Mong's work refers to the use of ACO algorithm in routing switches instead of OSPF or RIP algorithms. Empirical results using ACO in routing and load balance are encouraging [11].

Formal studies like Gutjahr's studies [9] shown that convergence of ACO based algorithms tend to one, but this study was done for a typical NP problem: travelling salesman problem.

A future work to this paper is to apply the algorithm in a fabric switched network [16], but it is not an easy task. Some simulations were done by Navarro and Sinclair [13] in generic networks, but could be interested to apply this modified algorithm based in ACO and MACO in storage area networks.

The repelling factor is the key for disjunctive paths if it is settled properly. If the value of are higher then disjunctive paths will be obtain in the final routing tables.

\section{ACKNOWLEDGMENTS}

Thanks to anonymous people who reviewed this paper and helped with valuable comments to formulate it.

\section{REFERENCES}

1. C, Blum. Beam-ACO hybridizing ant colony optimization with beam search: an application to open shop scheduling. Computers \& OR 32: 1565-1591 (Belgium, 2005).

2. Brocade web page. (October, 2005) http://www.brocade.com

3. A. Coloni, M.Y. Dorigo, V. Maniezzo. An investigation of some properties of an ant algorithm. (Proc. Parallel Problem Solving from Nature Conference PPSN`92, Brussels, Belgium, 1992) pp. 509-520. 
4. A. Coloni, M. Dorigo, V. Maniezzo. Distributed optimization by ant colonies. (Proc. First European Conf. On Artificial Life (ECAL'91), París, France, 1991) pp. 134-142.

5. M. Dorigo, V. Maniezzo, A. Coloni. The ant system: a cooperative learning approach to the travelling salesman problem. IEEE Trans. Evolutionary Computation, 1(1):1-13, (1996).

6. M. Dorigo, V. Maniezzo, A. Colorni. The Ant System: Optimization by a colony of cooperating agents. IEEE Transactions on Systems, Man, and Cybernetics-Part B, 26(1):29-41. (1996).

7. Fibre Channel Industry Association (FCIA) (October, 2005) http://www.fibrechannel.org

8. M. Fredman. An introduction to SAN Capacity Planning. Datacore Software Company.

htp:/www.demandtech.com/Resources/Papers/Intro\%20to\%20SAN\%20cap acity\%20planning.pdi (March, 2006)

9. W. Gutjahr. A generalized convergence result for the Graph-Based Ant System Metaheuristic. Future Generation Computer Systems. Vienna. (2000).

10. McDATA page. (October, 2005) http:/www mcdata.com

11. K. Mong, W. Hong. Ant Colony Optimization for Routing and Loadbalancing: Survey and New Directions. IEEE Transactions on systems, man, and cybernetics - Part A: Systems and Humans, (33):5. (September, 2003).

12. X. Molero, F. Silla, V. Santonia, J. Duato. Modeling and Evaluation of Fibre Channel Storage Area Networks. http:/esce.uark.edu-aapon/courses/ ioparallel/presentations/31.ppt España. (2005).

13. G. Navarro, M. Sinclair. Ant Colony Optimisation for Virtual-WavelengthPath Routing and Wavelength Allocation. (MACO) Univ. Of Essex. UK. (NASA). Proceedings of the Congress on Evolutionary Computation. (1999).

14. M. Reimann., M. Laumanns. A hybrid ACO algorithm for the Capacitated Minimum Spanning Tree Problem. ECAI2004. Workshop HYBRID METAHEURISTICS (HM 2004). (2004).

15. R. Schoonderwoerd, O. Holland, J. Bruten. Ant-like agents for load balancing in telecommunication networks. In: AGENTS '97: Proceedings of the first international conference on Autonomous agents, New York, NY, USA, ACM Press (1997), pp. 209-216.

16. Storage Networking Industry Association web page (SNIA) (October, 2005) http:/www.snia.org

17. T. Skie, O. Lysne, J. Flich, P. López, A. Robles, J. Duato. Lash-Tor: A generic transition-oriented routing algorithm. ICPADS 2004. (2004) pp. 595-604.

18. G. Di Caro, M. Dorigo. Two Ant Colony Algorithms for Best-Effort Routing in Datagram Networks. Proceedings of PDCS'98 - 10th International Conference on Parallel and Distributed Computing and Systems. (Las Vegas, Nevada, October 28-31, 1998).

19. M. Dorigo, T. Stuetzle. Ant Colony Optimization. MIT Press. ISBN 0-26204219-3. (2004). 